\title{
Papers
}

\section{Preferences for chemotherapy in patients with advanced non-small cell lung cancer: descriptive study based on scripted interviews}

\author{
Gerard Silvestri, Robert Pritchard, H Gilbert Welch
}

\begin{abstract}
Objective: To determine how patients with lung cancer value the trade off between the survival benefit of chemotherapy and its toxicities.

Design: Scripted interviews that included three hypothetical scenarios. Each scenario described the same patient with metastatic non-small cell lung cancer with an expected survival of 4 months without treatment. Subjects were asked to indicate the minimum survival benefit required to accept the side effects of chemotherapy in the first two scenarios (mild toxicity and severe toxicity). In the third scenario, subjects were asked to choose between chemotherapy and supportive care when the benefit of chemotherapy was either to prolong life by 3 months or to palliate symptoms.

Subjects: 81 patients previously treated with cis-platinum based chemotherapy for advanced non-small cell lung cancer.

Main outcome measure: Survival threshold for accepting chemotherapy.

Results: The minimum survival threshold for accepting the toxicity of chemotherapy varied widely in patients. Several patients would accept chemotherapy for a survival benefit of 1 week, while others would not choose chemotherapy even for a survival benefit of 24 months. The median survival threshold for accepting chemotherapy was 4.5 months for mild toxicity and 9 months for severe toxicity. When given the choice between supportive care and chemotherapy only $18(22 \%)$ patients chose chemotherapy for a survival benefit of 3 months; $55(68 \%)$ patients chose chemotherapy if it substantially reduced symptoms without prolonging life.

Conclusions: Patients' willingness to accept chemotherapy for the treatment of metastatic lung cancer varies widely. Many would not choose chemotherapy for its likely survival benefit of 3 months but would if it improved quality of life. The conflict between these patients' preferences and the care they previously received has several explanations, one being that some patients had not received the treatment they would have chosen had they been fully informed.
\end{abstract}

\section{Introduction}

Lung cancer is a common disease that is difficult to treat successfully. In the United States each year about 178000 people are diagnosed with lung cancer and about 160000 die of the disease, making it the leading cause of cancer related mortality. ${ }^{1}$ In the United Kingdom the death rate from lung cancer is similarly high, and it is even higher in eastern Europe and Russia. ${ }^{1}$ Most patients have non-small cell lung cancer and the majority of them have metastatic disease-either at the time the disease is diagnosed or during the course of their illness. ${ }^{2}$ Median survival is only about 4 months in untreated patients with metastatic non-small cell lung cancer. $^{3}$

Several meta-analyses concluded that chemotherapy is effective in the treatment of metastatic nonsmall cell lung cancer. ${ }^{3-5}$ The impact of chemotherapy on survival is limited, however; median survival is improved by about 1.5-3 months. ${ }^{3}$ Based on these data some authors advocate chemotherapy as standard treatment for all patients with metastatic non-small cell lung cancer. ${ }^{5}$ Other authors are more cautious in their interpretation in the absence of additional information on quality of life and the informed preferences of patients for the expected effects of treatment. ${ }^{34}$ Although most patients in the United Kingdom with metastatic non-small cell lung cancer are not considered for cytotoxic chemotherapy, many such patients are in the United States.

Although patient preferences have been systematically examined in breast cancer, ${ }^{6}$ little is known about how lung cancer patients value the potential benefits and risks of chemotherapy. Therefore, we assessed the treatment preferences of a group of patients who recently completed a course of chemotherapy for nonsmall cell lung cancer. We determined the minimum survival benefit necessary before they would accept a treatment regimen with its associated toxicity.

\section{Subjects and methods}

\section{Subjects}

All study subjects had advanced (stage III or IV) non-small cell lung cancer diagnosed histologically and had received at least one cycle of cis-platinum based chemotherapy (with or without radiotherapy).

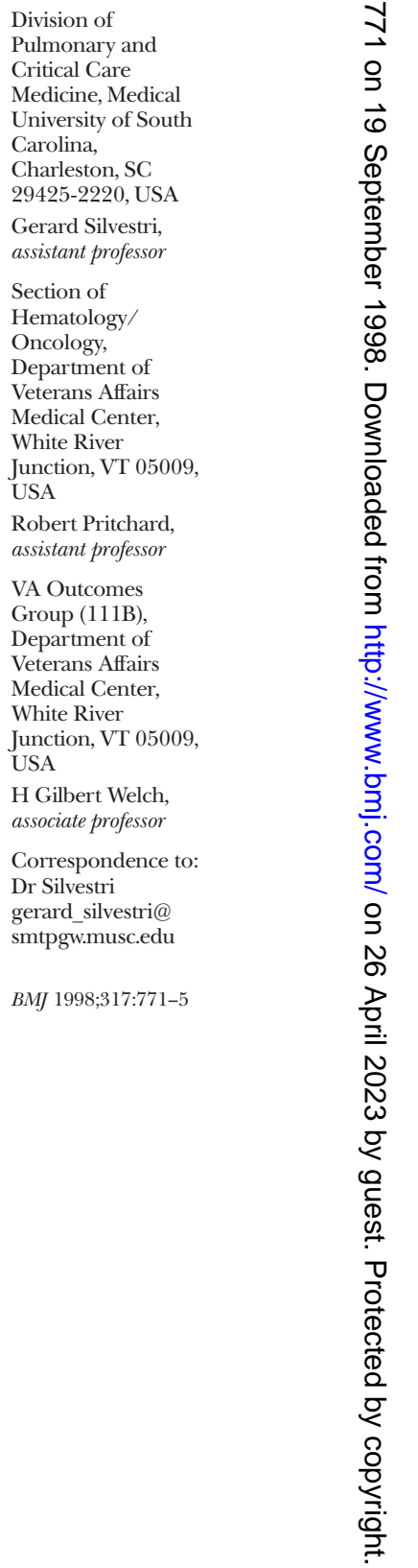


To ensure that responses were not influenced by recently experienced side effects, subjects were interviewed at least 1 month after completion of chemotherapy. Subjects with known or suspected brain metastases were excluded.

The subjects were recruited from three practice settings: the cancer centre at the Medical University of South Carolina, two department of veteran affairs medical centres (White River Junction, VT and Charleston, SC), and two community office practices (Hilton Head, SC and Charleston, SC).

\section{Interview structure}

Each interview followed the same format. Subjects were initially asked five questions about their understanding of treatment options before chemotherapy and their experience during chemotherapy. In addition they were asked to rate their overall quality of life during chemotherapy as either excellent, very good, good, fair, or poor. They were then given three scenarios, each describing the same hypothetical patient: a women with advanced metastatic lung cancer who has been told by her physician that she has an incurable illness and an expected survival without treatment of approximately 4 months. The scenarios were presented in the following order:

Scenario 1: mild toxicity-In this scenario chemotherapy was described as producing mild side effects and as being well tolerated. The side effects lasted several days after the treatment cycle and comprised nausea, fatigue, and occasional diarrhoea. The benefits of treatment were not discussed.

Scenario 2: severe toxicity - In this scenario chemotherapy was described as producing severe side effects and included the potential need for hospitalisation and a $1 \%$ chance of death. The side effects lasted several days after the treatment cycle, but were more numerous than in the first scenario: fatigue and weakness, poor appetite, mouth sores, diarrhoea, infection, and fever. Again, the benefits of treatment were not discussed.

Subjects were asked to choose the minimum survival benefit required to accept chemotherapy for the treatment of metastatic lung cancer in these two scenarios. As in previous studies of patient preferences for cancer treatment, a modification of the time trade off approach was used. ${ }^{78}$ Briefly, after hearing the scenario the subject was asked: "If you were this patient would you agree to this standard treatment if it added 1 week to your life?" If the answer was no the question was repeated substituting 1 month for 1 week. The process was repeated using different survival durations (3 months, 6 months, 12 months, 18 months, and 24 months) until the subject judged the benefit to be of sufficient importance to warrant the side effects.

Scenario 3: supportive care versus chemotherapy-In this scenario the benefit of treatment was made explicit. The hypothetical patient is offered the choice between supportive care (average survival 4 months) and chemotherapy (average survival 7 months). She is told that supportive care addresses a patient's comfort needs (and may include radiation) and that its goal is to alleviate pain and other symptoms associated with cancer. She is told about both the mild and severe side effects of chemotherapy and that her chance of experiencing severe side effects is $20 \%$. After this scenario the subject was asked: "If you were this patient which would

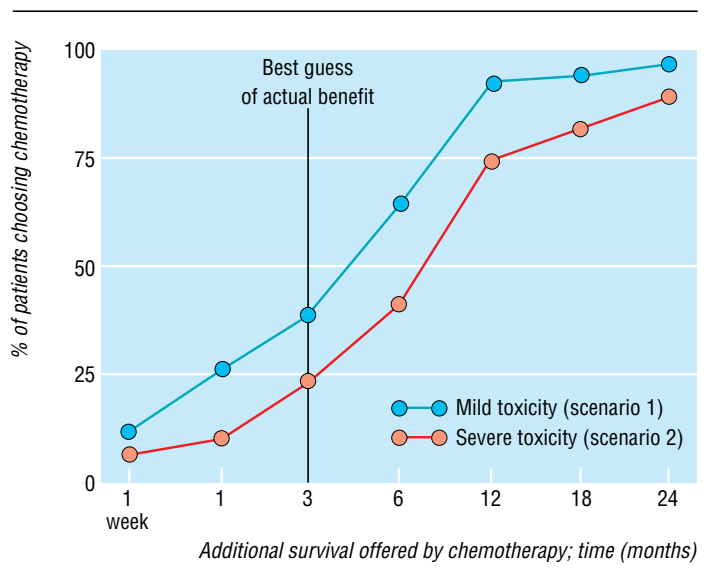

Fig 1 Treatment preferences for 81 patients who had had chemotherapy for lung cancer. Relation between additional survival offered and percentage of patients choosing chemotherapy is shown for mild toxicity (scenario 1) and severe toxicity (scenario 2)

you choose, supportive care or chemotherapy?" In addition, subjects were asked: "If you were this patient would you take this treatment if it did not prolong your life but significantly reduced the pain and other symptoms that might be related to your cancer?" Finally, to determine their willingness to be randomised to either supportive care or chemotherapy subjects were asked: "Would you allow a flip of the coin to determine which therapy you would receive?"

\section{Analysis}

For scenarios 1 and 2 we recorded the shortest survival duration at which each subject chose chemotherapy. We then constructed a cumulative distribution of the percentage of subjects choosing chemotherapy as a function of the additional survival offered by chemotherapy (fig 1).

Because we only provided seven discrete survival categories (1 week, 1, 3, 6, 12, 18, and 24 months) we were unable to determine precisely the survival duration at which each subject was indifferent to the choice presented. To estimate this threshold value for each subject we averaged the longest survival duration for which chemotherapy was rejected with the shortest survival duration for which chemotherapy was accepted. For example, if a subject rejected chemotherapy when offered 6 months' survival benefit and accepted chemotherapy when offered 12 months' survival benefit then the threshold value was 9 months. This survival threshold was used both to characterise patient subgroups (table) and to stratify the proportion choosing best supportive care in scenario 3 (fig 2).

Three subjects rejected chemotherapy even when offered 24 months survival benefit in the mild toxicity scenario (scenario 1), as did six subjects in the severe toxicity scenario (scenario 2). Because we did not ask about periods longer than 24 months we do not have the survival duration for which chemotherapy is acceptable in these individuals, and thus we are unable to estimate the threshold value. For the purposes of the statistical tests we arbitrarily assigned 24 months as the threshold value for these patients.

We used the Kruskal-Wallis test for the two nominal subgroups (sex, treatment setting) and the non- 
parametric test of trend for the two ordered subgroups (age, quality of life), to compare median survival thresholds (scenarios 1 and 2) across patient subgroups. We used the $\chi^{2}$ test for the nominal subgroups and $\chi^{2}$ test of trend for the ordered subgroups (including the subgroups in fig 2) for differences across patient subgroups in scenario 3. All analyses were performed with STATA 4.0 (College Station, TX).

\section{Results}

The 81 patients, all of whom had received chemotherapy for their lung cancer, agreed to discuss and therefore reconsider decisions for various survival durations. Although a few patients found the process difficult, all patients who started the survey were able to finish it. Generally we were able to solicit the responses to the three scenarios in less than 20 minutes per patient although longer discussions often ensued.

Figure 1 shows the treatment preferences for the 81 patients in the scenarios including side effects: mild toxicity (scenario 1) and severe toxicity (scenario 2 ). The figure is a cumulative distribution of the percentage of patients choosing chemotherapy as a function of the survival benefit offered. As expected, for any given level of survival a higher proportion of patients chose chemotherapy in the mild toxicity scenario.

Most surprising was the heterogeneity of the expressed preferences. In the setting of severe toxicity, for example, five $(6 \%)$ patients would choose chemotherapy for only 1 week's survival benefit while nine $(11 \%)$ would not choose the treatment even when offered 24 months' survival benefit. In both scenarios, however, less than half the patients would choose chemotherapy given the best guess of benefit-3 months' additional survival.

The table details the responses to the three scenarios and considers various patient subgroups. Overall, the median survival threshold for accepting chemotherapy was 4.5 months for mild toxicity and 9

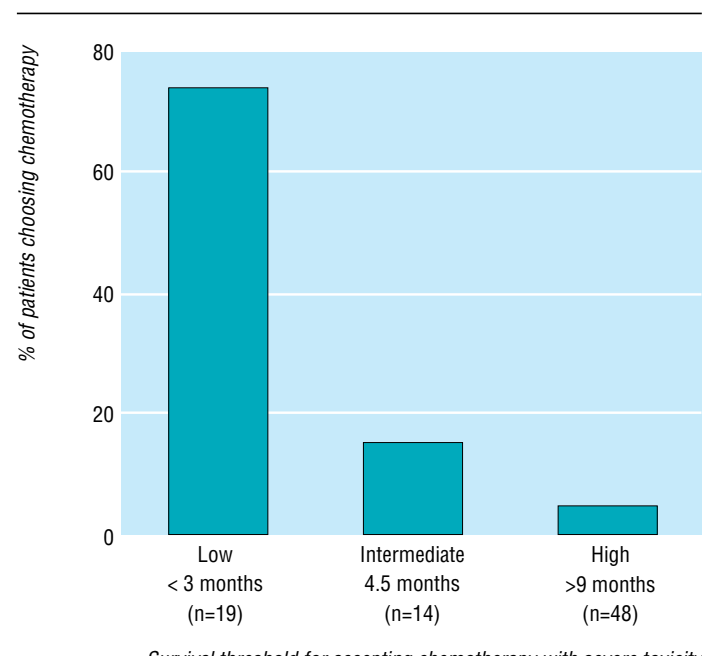

Fig 2 Percentage of patients choosing chemotherapy instead of supportive care (scenario 3) stratified by their imputed survival threshold for accepting chemotherapy with severe toxicity (scenario 2). ( $\chi^{2}$ test of trend $P<0.001$.) Overall, $78 \%$ of the patients chose supportive care
Responses to three scenarios by patient subgroups ( $P$ values are for differences across subgroups)

\begin{tabular}{|c|c|c|c|c|}
\hline \multirow[b]{2}{*}{ Patient subgroup } & \multirow[b]{2}{*}{$\begin{array}{c}\text { No }(\%) \text { of } \\
\text { patients }\end{array}$} & \multicolumn{2}{|c|}{$\begin{array}{l}\text { Median survival threshold for } \\
\text { accepting chemotherapy (months) }\end{array}$} & \multirow{2}{*}{$\begin{array}{c}\begin{array}{c}\% \text { of patients } \\
\text { choosing } \\
\text { supportive care }\end{array} \\
\text { Scenario } 3 \\
\end{array}$} \\
\hline & & $\begin{array}{c}\text { Scenario } 1 \\
\text { (mild toxicity) }\end{array}$ & $\begin{array}{c}\text { Scenario } 2 \\
\text { (severe toxicity) }\end{array}$ & \\
\hline All patients & $81(100)$ & 4.5 & 9.0 & 78 \\
\hline \multicolumn{5}{|l|}{ Age (years): } \\
\hline$<60$ & $31(38)$ & 2.0 & 4.5 & 65 \\
\hline $60-70$ & $30(37)$ & 4.5 & 9.0 & 87 \\
\hline$>70$ & $20(25)$ & 4.5 & 9.0 & 85 \\
\hline$P$ value & & 0.14 & 0.01 & 0.07 \\
\hline \multicolumn{5}{|l|}{ Sex: } \\
\hline Men & $54(67)$ & 4.5 & 9.0 & 74 \\
\hline Women & $27(33)$ & 4.5 & 9.0 & 85 \\
\hline$P$ value & & 0.23 & 0.32 & 0.28 \\
\hline \multicolumn{5}{|l|}{ Treatment setting: } \\
\hline University cancer centre & $29(36)$ & 9.0 & 9.0 & 86 \\
\hline Community practice & $29(36)$ & 2.0 & 9.0 & 72 \\
\hline Veterans' medical centre & $23(28)$ & 4.5 & 4.5 & 74 \\
\hline$P$ value & & 0.19 & 0.10 & 0.39 \\
\hline \multicolumn{5}{|c|}{ Self assessed quality of life during chemotherapy: } \\
\hline Excellent & $8(10)$ & 4.5 & 6.8 & 87 \\
\hline Very good & $23(28)$ & 4.5 & 9.0 & 70 \\
\hline Good & $22(27)$ & 3.3 & 4.5 & 68 \\
\hline Fair & $15(19)$ & 4.5 & 9.0 & 87 \\
\hline Poor & $13(16)$ & 9.0 & 15.0 & 92 \\
\hline$P$ value & & 0.01 & 0.01 & 0.31 \\
\hline
\end{tabular}

months for severe toxicity. As might be expected, elderly patients tended to demand greater benefit before accepting chemotherapy-a trend which was significant when severe toxicity was presented. Sex and treatment setting had little relation to these thresholds. There was, however, a significant trend with self assessed quality of life during chemotherapy: patients reporting lower quality of life during chemotherapy had higher thresholds for accepting chemotherapy.

When offered the choice between supportive care and chemotherapy (scenario 3), only 18 (22\%) patients chose chemotherapy. Figure 2 displays their responses stratified by the survival threshold imputed from scenario 2. As expected, patients with lower survival thresholds in scenario 2 were more likely to choose chemotherapy when offered a direct choice. Although only $18(22 \%)$ subjects chose chemotherapy for 3 months' improvement in survival the majority $(\mathrm{n}=55$, $68 \%$ ) would choose chemotherapy if it substantially reduced symptoms without prolonging life.

The patients had strong views on chemotherapy. One patient who chose chemotherapy for only 1 week's survival benefit theorised that the cure for lung cancer could be discovered during that week-he wouldn't want to miss the opportunity to be cured, however remote. Conversely, a second patient who did not choose chemotherapy even when offered 24 months' survival benefit said that she had lived a full and productive life and would not want anything to interfere with the quality of time she had left. The resolve of these patients was also evidenced by our finding that only $14(17 \%)$ were willing to be randomised between supportive care and chemotherapy.

\section{Discussion}

Patients previously treated with chemotherapy vary considerably in their attitudes towards this treatment 
for advanced lung cancer. For some patients, even 2 years' survival benefit did not constitute a fair trade off for the toxicities associated with chemotherapy. Other patients required very little, if any, increase in survival for chemotherapy to seem worthwhile. Most patients, however, expected more in return from chemotherapy than the best estimate of 3 months' survival benefit. Furthermore, when given the choice between chemotherapy and supportive care only a quarter of patients would choose chemotherapy if its benefit was only to prolong survival.

These results seem at odds with the care these patients previously received. All patients were treated with chemotherapy so why would only a quarter of them make the same decision again? This disparity may reflect either problems with our method for assessing patient preferences or problems in the initial decision making process.

How patient preferences are assessed clearly matters. Several methods are available for assessing patient preferences, including the time trade off and standard gamble. ${ }^{9}$ Although it is clear that different methods will often yield different results, ${ }^{10-12}$ it is less clear if any method accurately captures the patients' real attitudes toward different outcomes. ${ }^{13}$ We did not use the classic time trade off technique and our scenarios were not designed to elicit formal patient utilities for discrete health states. Rather, the scenarios were designed to be easily understood. We wanted to approximate what patients with metastatic lung cancer might be told by their physician and to allow a simple expression of their treatment preference. It is possible that our results would be different had we used a different method.

We are confident, however, that our approach for assessing patients' attitudes towards the trade off between survival and toxicity was at least understood by the patients. The patients' responses were consistent. Each patient's survival threshold either remained stable or increased as the toxicity of treatment increased. In other words, no patient chose chemotherapy in scenario 2 (severe toxicity) for less survival time than they did in scenario 1 (mild toxicity). And although patients may have not understood in scenario 3 that chemotherapy can include the services of supportive care there was a direct relation between the survival thresholds imputed from the first two scenarios and the choice of treatment in scenario 3 .

The correct units for expressing the benefits of treatment may also matter. In our scenarios the benefits were expressed as an average gain in survival. And while it is true that for most patients chemotherapy improves survival in metastatic nonsmall cell lung cancer by no more than several months in the majority of patients, the use of averages may hide the fact that a few patients may benefit substantially. Since the framing of benefits can have important consequences on the choice of treatment ${ }^{14}$ it is possible that if we expressed the benefits of treatment differently we would have got different results. Whether the expression of a small chance of a big gain would have meaningfully changed our results is the subject of future investigation.

There may be other information besides toxicity, survival, and quality of life that patients with terminal illnesses consider important when thinking about complex decisions. For example, fear of alienating family members or acting to maximise the chance of reaching some future landmark event (for example, a son or daughter's wedding) may overshadow quantitative estimates of survival. These types of considerations are not easily dealt with in our scenario based decision making.

There may also be problems in the initial decision making process. Firstly, patients may have been unaware that there was a choice between supportive care and treatment with chemotherapy. Only a quarter of our subjects could recall having supportive care discussed as an option when they were originally diagnosed with advanced lung cancer. Secondly, it is possible that the patients were not properly informed about the risks and survival benefits of chemotherapy-either because the benefits of chemotherapy were portrayed as overly optimistic or because patients misunderstood or chose to ignore information about the expected size of the survival benefit. We did not, however, interview patients at the time of the initial decision. Had we done so our results may have been different. ${ }^{15}$ Alternatively our results may simply reflect the patients' recent experience with chemotherapy-"knowing what I know now, I wouldn't make the same choice."

\section{Implications}

Our results have several important implications. The finding that most patients would not choose chemotherapy for a survival benefit of 3 months suggests that from the patient's perspective best supportive care is a realistic treatment option worthy of discussion. Yet there was also striking variation in the patients' willingness to accept cancer treatment that was potentially toxic. This finding, which has been seen in similar studies, ${ }^{815}{ }^{16}$ reinforces the notion that for patients there is no right or wrong answer. When different patients value alternative treatment options differently, clinical rules or protocols based on average preferences will not be effective in helping patients select the treatment they actually want. ${ }^{13}$ And while guidelines may be useful tools for synthesising the scientific evidence used to formulate clinical decisions they cannot obviate the need for good compassionate clinical decision making that is responsive to patients' values.

But patients also care about more than survival when faced with difficult choices. Although most of the patients who participated in this study would not choose chemotherapy for a survival benefit of 3 months the majority would if it improved the symptoms of their cancer. Adequately informing patients about the benefits of chemotherapy therefore requires better information about the impact of chemotherapy, compared with an untreated control group, on the quality of life of patients with advanced lung cancer. It also requires a better understanding of what other information is important to patients facing these types of decisions.

\section{Conclusion}

In 1997, between 80000 and 100000 patients in the United States will have to decide about having chemotherapy for the treatment of advanced non-small cell lung cancer. Because the survival benefit is modest and the toxicities real, the decision is extremely difficult. Choosing the proper treatment for cancer patients 
Key messages

- The median survival of patients with metastatic non-small cell lung cancer is improved by about 3 months with the addition of chemotherapy

- Lung cancer patients who had had chemotherapy were interviewed in this study to learn about their treatment preferences with a range of survival benefits

- Several patients would choose chemotherapy for a survival benefit of as little as 1 week, while others would not choose chemotherapy even when offered a survival benefit of 24 months

- Most patients would not choose chemotherapy for its likely survival benefit of 3 months, but would if it improved quality of life

- Some patients with lung cancer may not be getting the treatment they would choose were they fully informed

requires that they are fully aware of the merits of chemotherapy. Our results suggest that some patients may not be getting what they want.

We thank Amanda Budak for helping with the survey, Robert Nease for helping with its design, Gary Thomas, George Geils, Robert Wall, and David Ellison for the use of their surgeries, and the patients. The views expressed in this paper are not necessarily those of the Department of Veterans Affairs or the United States government.

Contributors: GS and RP conceived and designed the study, conducted the interviews, and wrote the paper. HGW designed the study, analysed the data, and wrote the paper; he will act as guarantor for the paper.

1 Parker S, Tong T, Bolden S, Wingo P. Cancer statistics, 1997. CA Cancer J Clin 1997; 47:5-27.

2 Ginsberg R, Kris M, Armstrong J. Cancer of the lung: non-small cell lung cancer. In: DeVita JV, Hellman S, Rosenberg S, eds. Cancer:principles and practice of oncology. Philadelphia: JB Lippincott, 1993:673-723.

3 Grilli R, Oxman A, Julian J. Chemotherapy for advanced non-small cell lung cancer: how much benefit is enough? J Clin Oncol 1993;11:1866-72.

4 Marino P, Pampallona S, Preatoni A, Cantoni A, Invernizzi F. Chemotherapy vs supportive care in advanced non-small cell lung cancer: results of a meta-analysis of the literature. Chest 1994;106:861-5.

5 Souquet PJ, Chauvin F, Boissel JP, Cellerino R, Cormier Y, Ganz PA, et al Polychemotherapy in advanced non-small cell lung cancer: a meta-analysis. Lancet 1993;342:19-21.

6 Richards MA, Ramirez AJ, Degner LF, Fallowfield LJ, Maher EJ, Neuberger J. Offering choice of treatment to patients with cancer: a review based on symposium held at the 10th annual conference of the British Psychosocial Oncology Group, December 1993. Eur J Cancer 1995;31A:112-6.

7 Yellen S, Cella D, Leslie W. Age and clinical decision-making in oncology patients. J Natl Cancer Inst 1994;86:1766-70.

8 McQuellon RP, Muss HB, Hoffman SL, Russell G, Craven B, Yellen SB Patient preferences for treatment of metastatic breast cancer: a study of women with early breast cancer. J Clin Oncol 1995;13:858-68.

9 Sox H, Blatt M, Higgins M, Marton K. Medical decision making. Boston: Butterworths, 1988 .

10 Read J, Quinn R, Berwick D, Finebergh HF, Weinstein M. Preferences for health outcomes. Comparison of assessment methods. Med Decis Making 1984;4:315-29.

11 Nord E. Methods for quality adjustment of life years. Med Decis Making 1992;34:559-69.

12 Stiggelbout AM, Kiebert GM, Kievit J, Leer JW, Stoter G, de Haes JC. Utility assessment in cancer patients: adjustment of time tradeoff scores for the utility of life years and comparison with standard gamble scores. Med Decis Making 1994;14:82-90.

13 Kassirer J. Incorporating patients' preferences into medical decisions. $N$ Engl J Med 1994:330:1895-6.

14 McNeil B, Pauker S, Sox H, Tversky A. On the elicitation of preferences for alternative therapies. N Engl J Med 1982;306:1259-62.

15 Slevin ML, Stubbs L, Plant HJ, Wilson P, Gregory W, Armes PJ, et al. Attitudes to chemotherapy; comparing views of patients with cancer with those of doctors, nurses, and general public. BMJ 1990;300:1458-60

16 Brundage MD, Davidson JR, Mackillop WJ. Trading treatment toxicity for survival in locally advanced non-small cell lung cancer. J Clin Oncol 1997;15:330-40.

(Accepted 2 June 1998)

\section{Working hours as a risk factor for acute myocardial infarction in Japan: case-control study}

Shigeru Sokejima, Sadanobu Kagamimori

\author{
Abstract \\ Objective: To clarify the extent to which working \\ hours affect the risk of acute myocardial infarction, \\ independent of established risk factors and \\ occupational conditions. \\ Design: Case-control study. \\ Setting: University and general hospitals and routine \\ medical examinations at workplaces in Japan. \\ Subjects: Cases were 195 men aged 30-69 years \\ admitted to hospital with acute myocardial infarction \\ during 1990-3. Controls were 331 men matched at \\ group level for age and occupation who were judged \\ to be free of coronary heart diseases at routine \\ medical examinations in the workplace. \\ Main outcome measures: Odds ratios for myocardial \\ infarction in relation to previous mean daily working \\ hours in a month and changes in mean working \\ hours during previous year. \\ Results: Compared with men with mean working \\ hours of $>7-9$ hours, the odds ratio of acute \\ myocardial infarction (adjusted for age and
}

occupation) for men with working hours of $>11$

hours was 2.44 (95\% confidence interval 1.26 to 4.73$)$

and for men with working hours of $\leqslant 7$ hours was 3.07 (1.77 to 5.32). Compared with men who experienced an increase of $\leqslant 1$ hour in mean working hours, the adjusted odds ratio of myocardial infarction for men who experienced an increase of $>3$ hours was 2.53 (1.34 to 4.77 ). No appreciable change was observed when odds ratios were adjusted for established and psychosocial risk factors for myocardial infarction.

Conclusion: There was a $\mathrm{U}$ shaped relation between the mean working hours and the risk of acute myocardial infarction. There also seemed to be a trend for the risk of infarction to increase with greater increases in mean working hours.

\section{Introduction}

The occurrence of disease that may relate to working hours is an important consideration in Japan, where working hours have been unusually long. ${ }^{1}$ Uehata has 